\title{
Clinical Evaluation of Herbal Medicine (ICH-012) in Treating Acute Cerebral Haemorrhage: Safety and Efficacy from 6- to 72-Hour Time Window (CRRICHTrial-II)
}

\author{
Qixin Zhang, Liling Zeng, Xiuyan Chen, Yuexiang Zhou, \\ Baoying Gong, Haijun Li, and Jianwen Guo
}

Brain Center, The Guangdong Provincial Hospital of Chinese Medicine, The Second Teaching Hospital of Guangzhou University of Chinese Medicine, China

Correspondence should be addressed to Jianwen Guo; 306247680@qq.com

Qixin Zhang and Liling Zeng contributed equally to this work.

Received 22 February 2018; Revised 20 June 2018; Accepted 9 July 2018; Published 26 August 2018

Academic Editor: Claudia Di Giacomo

Copyright (c) 2018 Qixin Zhang et al. This is an open access article distributed under the Creative Commons Attribution License, which permits unrestricted use, distribution, and reproduction in any medium, provided the original work is properly cited.

\begin{abstract}
Background. Hypertensive intracerebral haemorrhage $(\mathrm{HICH})$, which is characterized by rapid change, high morbidity, and mortality, is extremely dangerous. Both medical and surgical treatments lack definitive evidence and remain controversial. A prospective RCT that we have conducted has shown that the usage of the herbal medicine ICH- 012 within $6 \mathrm{~h}$ of the event may increase the risk of haematoma enlargement and gastrointestinal bleeding. However, the volume of haematoma remains stable after $6 \mathrm{~h}$. Thus, we will increase the time window to the period from 6 to $72 \mathrm{~h}$ after onset to evaluate the safety and efficacy of ICH-012 treating ICH (ClinicalTrial.gov ID: NCT03354026). Methods/Design. The CRRICHTrial-II study, a prospective, doubleblinded, controlled, multicentre RCT, includes three groups: A, B, and C. Group A patients were treated with 8 herbal medicines (with 2 herbal medicines of Hirudo and Tabanus as well as 6 other combined herbal medicines of Group B) and Group C were placebo. Patients should meet all the inclusion criteria: age between 18 and 80 and diagnosis of HICH by brain CT scan between 6 and $72 \mathrm{~h}$ from the onset. The CT scan will be taken at four critical time points: baseline, between 6 and $72 \mathrm{~h}, 24 \mathrm{~h}$ after onset, and between 10 and 14 days after onset. The drug intervention lasts 10 days, and there is a follow-up visit taken after 90 days. The haematoma enlargement after $24 \mathrm{~h}$ onset as demonstrated by CT is the primary outcome. Discussion. A large amount of data from high-quality RCTs is needed for the extensive clinical application of herbal medicine. The CRRICHTrial-II will evaluate the safety and effectiveness of ICH-012 in a safer time window between 6 and $72 \mathrm{~h}$ and investigate the possible mechanisms of action and direction of herbal medicine in the haematoma growth after HICH. Trial registration at ClinicalTrial.gov, ID: NCT03354026, is registered on 23rd Nov. 2017.
\end{abstract}

\section{Update}

1.1. Background. Cerebrovascular disease has become the first leading cause of death in Chinese people, and haemorrhagic stroke (intracerebral haemorrhage, ICH) is one of the most important manifestations [1]. Prevention and standardized treatment are critical issues worldwide because of the rapid onset, rapid progress, high morbidity, and rates of disability for ICH $[2,3]$. It is generally believed that the enlargement of haematoma after ICH is an important factor of poor prognosis $[4,5]$. Moreover, as an independent risk factor for stroke prognosis, the enlargement of haematoma is shown to be closely related to some clinical indicators including the presence and number of CT spot sign number, hypertension, and coagulopathy [6-8]. In recent years, the genotype of ApoE 2 has been discovered to be related to the rupture of vessel walls in amyloid cerebrovascular disease as well as the haematoma enlargement of lobar haemorrhage; however, genetic testing to predict haematoma enlargement is still in the exploratory stage [9-11]. Unfortunately, neither medical nor surgical clinical trials have had positive results for the HICH treatment. Thus, we have no medications or 
surgical methods to reduce the mortality and morbidity of HICH patients [12-14].

In China, the curative effects of the traditional Chinese medicines for ICH have been demonstrated clinically, which plays an important role in reducing the mortality and disability of ICH. Herbal medicine that promotes blood circulation and reducing blood stasis was most commonly used in clinical practice [15-17]. On the other hand, according to research we have conducted a prospective, randomized, double-blind, controlled clinical trial (Trial registration clinicaltrials.gov: NCT01918722) in which the herbal medicine ICH-012 may increase the risk of haematoma growth and gastrointestinal bleeding without efficacy in reducing mortality or morbidity during the $6 \mathrm{~h}$ time window [18]. We may delay the time window of the drug delivery to 6-72 h onset at the next stage in consideration of the high incidence rate of haematoma enlargement within $6 \mathrm{~h}$. Furthermore, we must increase the sample size to more than 360 individuals.

\section{Methods/Design}

2.1. Objectives. This trial's primary aim is to evaluate the safety and efficacy of the herbal medicine ICH-012 in treating $\mathrm{AICH}$ within the time window of 6 to $72 \mathrm{~h}$ from onset. Exploring the possible mechanisms of action of the herbal medicine is also our concern.

2.2. Study Design. This is a prospective, randomized, double-blinded, controlled trial (ClinicalTrial.gov, ID: NCT03354026) involving 7 participating neurological centres. The flowchart of this research is shown in Figure 1. Patients with acute intracerebral haemorrhage diagnosed by brain CT scan within 6 to $72 \mathrm{~h}$ onset are included in the scope of screening first. The informed consent will be signed when the patient meets all the inclusion criteria shown in the following category of Eligibility Criteria. The trial includes three random allocation groups: Groups A, B, and C. All the enrolled patients will take the first dose of study medicine within 6 to $72 \mathrm{~h}$ of ictus before scoring NIHSS and GCS. The medicine intervention will last 10 days, while the CT scan will be taken at three critical time points: between 6 and 72 h, $24 \mathrm{~h}$ after, and between 10 and 14 days after. There is a follow-up visit taken after 90 days, during which the researchers will finish the questionnaires and summary table. The primary outcome is haematoma enlargement after $24 \mathrm{~h}$ onset demonstrated by CT, while the secondary outcomes include mortality and severe adverse events.

This trial will be conducted in the departments of neurosurgery at the following 7 Chinese hospitals: (1) The Second Teaching Hospital of Guangzhou University of Chinese Medicine, Guangzhou; (2) Lianjiang People's Hospital, Lianjiang; (3) Shenyang Second Hospital of Traditional Chinese Medicine, Shenyang; (4) The Third People's Hospital of Hubei Province, Hubei; (5) Liaocheng People's Hospital, Liaocheng; (6) Zengcheng Hospital of Traditional Chinese Medicine, Zengcheng; and (7) Cong Hua Hospital of Chinese Medicine, Conghua. Researchers from different hospitals will receive unified training and assessment before the beginning of the trial. Quality control of the trial will be undertaken by the subject team at least once a year.

\section{Eligibility Criteria}

\subsection{Inclusion Criteria}

(1) Men and women aged from 18 to 80 years

(2) Acute cerebral haemorrhage confirmed by brain CT scan within 6 to $72 \mathrm{~h}$ from onset

(3) Glasgow Coma Scale $\geq 6$

(4) Signed informed consent

\subsection{Exclusion Criteria}

(1) Secondary cerebral haemorrhage caused by brain tumour, blood diseases, cerebrovascular malformation (anomaly), aneurysm, or other pathology

(2) Patients with severe heart, liver, and renal insufficiency

(3) Intolerance to traditional Chinese medicine (TCM) or history of allergies

(4) Patients with severe cerebral hernia in the early onset stage

(5) Poor compliance

3.3. Centre Eligibility. A total of 7 hospitals across China participated in this study, including the principal responsible unit. All the participation centres are equipped with qualified neurology centres, equipment, and specialists for standardized medical care and clinical trials.

\section{Sample Size and Randomization}

4.1. Sample Size. The enlargement percentage of the haematoma volume measured on the CT scan at the time $24 \mathrm{~h}$ after onset is the primary outcome of this study. We designed 3 groups (A, B, and C) to balance and calculate the enlargement percentage of the haematoma by blinded methods. The hypothesis is that haematoma in the three groups is not different. According to a previous literature review, the rate of haematoma enlargement at $24 \mathrm{~h}$ after onset is likely between $14 \%$ to $20 \%$ [19-21]. The Chinese herbal medicines used in treating $\mathrm{AICH}$ in the 3 groups associated with the trial are shown in Table $1[17,18]$. With the parameters set at $\alpha=0.05,1-\beta=80 \%$, and the primary outcome rate $=20 \%$, we used the following formula to calculate the sample size. The loss rate is supposed to be approximately $10 \%$, and 360 patients are necessary.

$$
\begin{array}{r}
\mathrm{n}=\frac{1641.6 \gamma}{\left(\sin \bigwedge(-1) \sqrt{ }\left(\pi \_\max \right)\right)-\sin \wedge(-1) \sqrt{ } \pi \_\min \wedge 2} \\
\left(\gamma \text { is } 12.65, \pi \_\max 38 \% \text { and } \pi \_\min 7 \%\right)
\end{array}
$$

4.2. Randomization. The Chinese medical herbs used here are from the Pharmacopoeia of the People's Republic of China released on 5 June 2015. Our randomized programme was completed by the key clinical research laboratory of the Traditional Chinese Medicine Hospital of Guangdong 


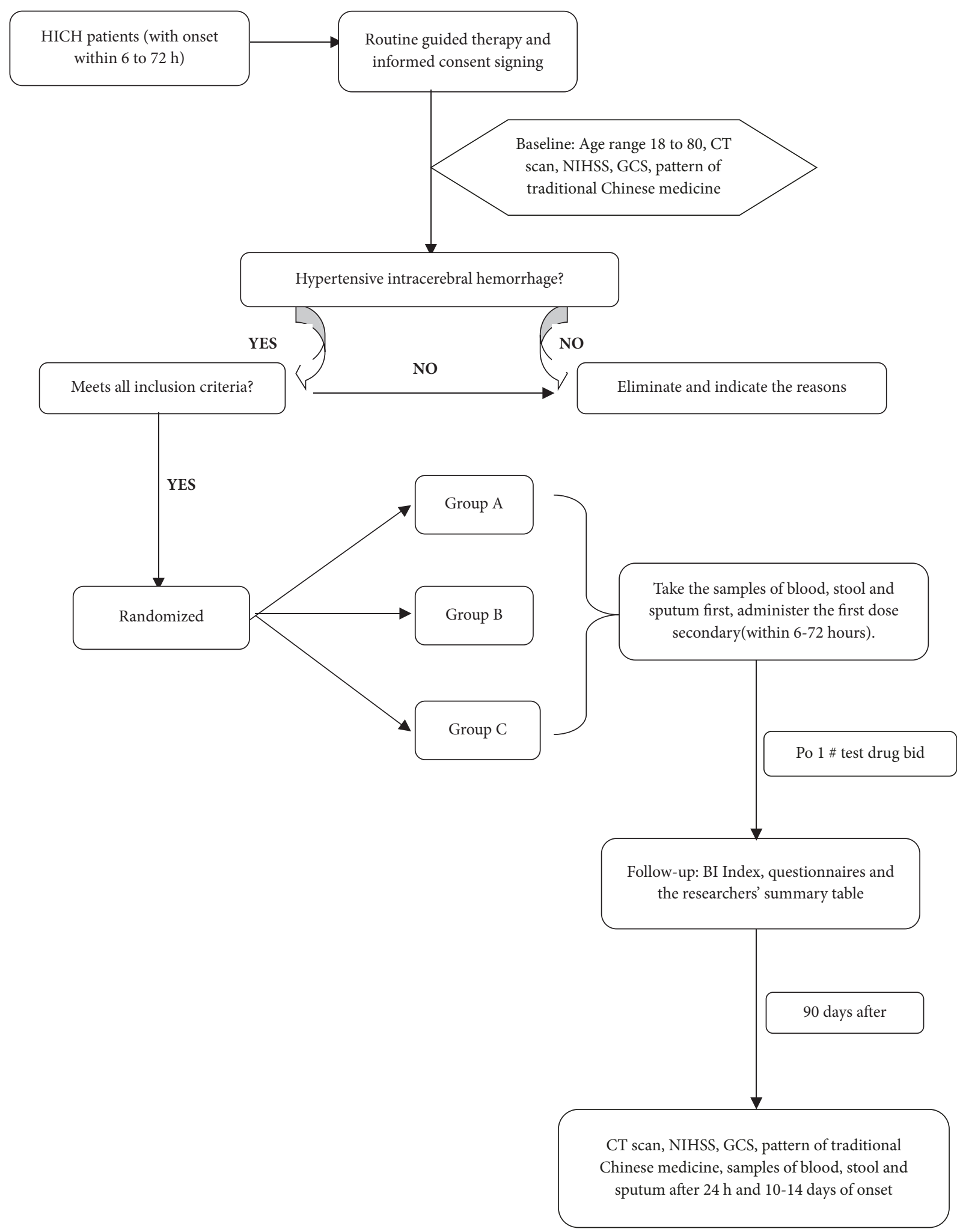

FIgure 1: The flowchart of the CRRICHTrial-II. 
TABLE 1: The univariate analysis on the haematoma enlargement (\%)[17].

\begin{tabular}{lcccc}
\hline Factor & $\begin{array}{c}\text { Haematoma } \\
\text { enlargement } \\
(\%)(\mathrm{n}=43)\end{array}$ & $\begin{array}{c}\text { Non- } \\
\text { haematoma } \\
\text { enlargement } \\
(\%)(\mathrm{n}=213)\end{array}$ & Value & $\mathrm{p}$ \\
\hline PBC and RBS herbal & & & & \\
$\quad$ Not used & $19(44.2)$ & $78(36.6)$ & & \\
PBC & $2(4.7)$ & $26(12.2)$ & 0.181 & 0.149 \\
RBS & $22(51.2)$ & $109(51.2)$ & & \\
PBC and RBS & $24(55.8)$ & $135(63.4)$ & 0.870 & 0.390 \\
Leech & $22(51.2)$ & $109(51.2)$ & 0.00 & 1.00 \\
Leonurus & $24(58.1)$ & $124(58.2)$ & 0.085 & 0.866 \\
Rhizoma & $22(51.2)$ & $109(51.2)$ & 0.00 & 1.00 \\
\hline
\end{tabular}

Province. We assigned 306 cases into three groups: Groups $\mathrm{A}, \mathrm{B}$, and $\mathrm{C}$ in the proportion of 1:1:1. Group A, the first experimental group, used RBS, which includes 8 herbal medicines, Hirudo and Tabanus, and 6 herbals of promoting blood circulation. Group B, the second experimental group, used an herbal medicine, which includes all the herbals in Group A except Hirudo, Tabanus, and rhubarb. Group C is a placebo group with dextrin, farina, and so on. This study adopts a stratified random sampling method and intraslice randomization on the basis of using PROC PLAN progress in SAS V9.2. In addition, the experimental measures and the control measures will be double blind. The surface of the opaque randomization envelopes will indicate the information of the test name, hospital name, and the entry sequence number of the patient. The research process of incorporating the patient, dispensing medicine depending on the random envelopes, and others will be supervised by the researchers.

\section{Intervention Treatment}

The first dose will be arranged within 6 to $72 \mathrm{~h}$ of onset, and the medicine will be taken twice a day for 10 days. The details of the treatment intervention in the three groups are shown in Table 2. All our pharmaceutical preparations are produced by the Kang Yuan pharmaceutical company, which has qualified pharmaceutical production facilities in China. Usage of the medicine is as follows: dissolve each bag of medicine in 80 to $100 \mathrm{ml}$ warm water and take orally or receive through nasal feeding by the researchers. Each time the medicine is administered, the researchers must record it and implement quality control.

5.1. CT Scanning. The first CT scan should be completed immediately after admission to identify $\mathrm{ICH}$, which may be less than $6 \mathrm{~h}$ or between 6 and $72 \mathrm{~h}$ from onset. The secondary CT scan must be done after the onset time at $24 \mathrm{~h}$, mainly to evaluate the haematoma growth and the oedema around the haematoma. The last CT scan can be completed within 10 to 14 days after onset, mainly to evaluate the enlargement or absorption of the haematoma. We define the amount of bleeding as the volume of the haematoma by the chef formula (the volume of haematoma=the maximum diameter of haematoma $\times$ wide diameter $\times$ CT dimension $\times$ CT layer thickness $\times \pi / 6$ ).

5.2. Follow-Up and Outcome Evaluation. We will set up 4 main study visit time points in this study to evaluate the patient's status dynamically. The 4 study visit time points include the following: when the subject is admitted, $24 \mathrm{~h}$ after onset, 10 to 14 days after onset, and 90 plus-or-minus 7 days after onset. The researchers must finish the GCS scoring, NIHSS scoring, water drinking test, traditional Chinese medicine pattern assessment form for stroke, etc. The BI index scoring and mRS scoring will be taken at the time point 10 to 14 days after onset and 90 plus-or-minus 7 days after onset. The water drinking test will be administered at all time points except for the first. Table 3 shows all the detailed information of the follow-up and outcome evaluation.

5.3. Primary Outcome. The enlargement percentage of the haematoma measured on the CT scan at the time $24 \mathrm{~h}$ after onset is one of the primary outcomes in this study. The definition of haematoma enlargement is a haematoma volume enlarged $33 \%$ or increased by $12.5 \mathrm{ml}$, which is compared with the two CT scans (6-72 $\mathrm{h}$ from onset and 24 $\mathrm{h}$ after onset or 6-72 $\mathrm{h}$ from onset and 10-14 days after onset)

5.4. Secondary Outcomes. There are 7 secondary outcomes: (1) GCS scoring scale for consciousness assessment; (2) NIHSS scoring scale for neurological impairment assessment; (3) BI index for living ability assessment; (4) social function activity questionnaire (FAQ) to evaluate the quality of life after stroke; (5) the case fatality rate on 14 days after the onset; (6) the disability rate at the 90 day follow-up; and (7) other general questionnaires to collect information about the blood type and genotype of the patients.

5.5. Adverse Event Validation. We record all adverse events during the experiment process in detail, and the adverse events should not be considered as a single symptom. The researchers must assign adverse events into three categories: mild, moderate and severe. Also, the possible causes and the processing method should be written on the case observation chart. If it is uncertain whether the symptom is related to the medicine, it will be recorded in detail. To judge the adverse reactions in clinical trials, we mainly follow these five principles: (1) the rationality of the time sequence of medication and adverse events; (2) adverse events reported of this herbal medicine in the past; (3) the withdrawal effects of this herbal medicine; (4) the results of the remedication after the disappearance of adverse events; and (5) considerations of other confounding factors. Lastly, the researchers will judge and decide the adverse event validation using descriptions of irrelevant, may not be relevant, probably relevant, most likely relevant, and certainly relevant.

5.6. Blinding. Before unblinding, only the researchers responsible for the randomized method knew the background grouping data. All the participants in this 
TABLE 2: Groups and Interventions.

\begin{tabular}{lcc}
\hline Groups & Assigned Interventions & Dosage and Taking \\
\hline ICH-012 & 8 herbal medicine (with 2 herbals for activating blood stagnation and expelling & One bag, po., bid for 10 \\
& blood stasis herbs) & days. Open the medicine \\
ICH-012-II & 6-herbal medicine (the same as ICH-012 but without 2 herbals- folium sennae & bag and take it after mixing \\
Placebo & and Snakegourd seed) & with 50-80 ml warm water \\
& Placebo medicine made of starch, bitter-tasting additive, and cyclodextrin & (Or take by nasal feeding). \\
\hline
\end{tabular}

TABLE 3: The flow table of the follow-up and outcome evaluation of clinical trial.

\begin{tabular}{|c|c|c|c|c|c|}
\hline Objects & Screening form & $\begin{array}{c}\text { Visit of 6-72h } \\
\text { after onset }\end{array}$ & $\begin{array}{c}\text { Visit of } 24 \mathrm{~h} \text { after } \\
\text { onset }\end{array}$ & $\begin{array}{c}\text { Visit after onset } \\
10 \text { to } 14 \text { days } \\
\end{array}$ & $\begin{array}{c}\text { Visit after onset } \\
90 \pm 7 \text { days }\end{array}$ \\
\hline Demographic data & $\sqrt{ }$ & & & & \\
\hline Medical history & $\sqrt{ }$ & & & & \\
\hline Physical examination & $\sqrt{ }$ & $\sqrt{ }$ & & & \\
\hline CT and CTA & $\sqrt{ }$ & $\sqrt{ }$ & $\sqrt{ }$ & $\sqrt{ }$ & \\
\hline Determination of inclusion or not & $\sqrt{ }$ & & & & \\
\hline Informed consent & $\sqrt{ }$ & & & & \\
\hline Biological specimen & $\sqrt{ }$ & $\sqrt{ }$ & $\sqrt{ }$ & $\sqrt{ }$ & \\
\hline Adjoint pharmacy and treatment & $\sqrt{ }$ & $\sqrt{ }$ & $\sqrt{ }$ & $\sqrt{ }$ & \\
\hline GCS, NIHSS, water drinking test & $\sqrt{ }$ & $\sqrt{ }$ & $\sqrt{ }$ & $\sqrt{ }$ & \\
\hline mRS, BI index and questionnaire & & & $\sqrt{ }$ & $\sqrt{ }$ & \\
\hline Adverse event & & $\sqrt{ }$ & $\sqrt{ }$ & $\sqrt{ }$ & $\sqrt{ }$ \\
\hline Summary of the subjects and researches & & & & & $\sqrt{ }$ \\
\hline
\end{tabular}

study, including the principal investigator, researchers, experimental subjects, controllers, and data analyst, will be blinded about the background data. Only in the case of severe adverse events closely related to the treatment will the blind be broken.

5.7. Statistical Analyses. The data analysis for this experiment is a comparative analysis of multiple independent sample rates, in accordance with the standard that $\alpha=0.05$ (the size of the first error in hypothesis testing), $1-\beta=0.9$ (the efficiency of the expected test), and $\delta=2$ (the significant differences, more than $50 \%$ of the volume of haematoma and greater than that by $2 \mathrm{ml}$ ). In this study, the percentage change in the volume of the haematoma is measured and calculated from the baseline CT scan and the $24 \mathrm{~h}$ CT scan. The 7 test centres, researchers, and the $\mathrm{CT}$ readers are fitted with a random effect model. Also, the baseline CT scan, time course from onset to CT scan, and interventions during the research are fitted with a fixed effect model. The percentage change rate of haematoma volume will be converted to logarithmic form and normally distributed. The two treatment groups will be analysed comparatively by chi-square distribution with Bonferroni correction.

5.8. Data Monitoring and Quality Control. The Data Safety Management Centre (DSM) from the Guangdong Province Hospital of Traditional Chinese Medicine is responsible for the data and safety monitoring. The data inspectors trained by the DSM will complete and submit the physical and chemical examination normal value range table to the DSM.
If there is any indication of impropriety, the DSM will stop the trial and the person responsible for the trial must make a detailed description. Annual quality control meetings will be held in the duty hospital, and the researchers from all the participating hospitals must give a report about the trial progress in detail. The patients joining the trial will be registered by key information on the ClinicalTrial.gov online website.

\section{Discussion}

Considering the lack of studies in TCM meeting the standard of RCT, further high-quality RCTs are required [22]. We have proven that the use of herbal medicine would not lead to the enlargement of haematoma within $24 \mathrm{~h}$ after onset in a retrospective study in 2015[17], which supports the traditional Chinese therapy of activating blood circulation and removing blood stasis in treating haemorrhagic stroke $[15,16]$. However, a prospective, randomized, open, doubleblind controlled clinical trial we have conducted previously showed the dangers of using PBS and RBS, which may lead to the enlargement of haematoma within $6 \mathrm{~h}$ onset [18]. The past surveys showed that the haematoma became stable and the rate of rebleeding was small $[19,20]$. Taking this into account, we speculate that it is safer to use herbal medicines for activating blood circulation and removing stasis after 6 $\mathrm{h}$ following onset. Thus, we designed a clinical trial about this herbal medicine as an intervention for acute intracerebral haemorrhage $(\mathrm{AICH})$. We expect to provide a new treatment that will be effective at reducing the fatality and disability 
of AICH under more rigorous preconditions. The CRRICHI study has been completed, and we have maintained good partnerships between all cooperative hospitals as well as developing rich experience with this kind of clinical trial. In all, these partnerships guarantee a good foundation for the CRRICH-II study. In this study, we suggested that the probable reason may be a small sample size for effective case analysis, the limitation of the time window, and the difference of the individuals' genotype or serum markers. With the development of precision medical treatment, some specific serum markers and some genotypes have been shown to be risk factors for haematoma enlargement [23]. At present, APOE genotype and von Willebrand factor genotype have been confirmed to be related to the enlargement of ICH haematoma $[10,24,25]$. The question of whether the individual sensitivity to traditional Chinese medicine is related to the serum markers or genotype requires further study. Further defining the relationship between serum markers and genotype will further expand the scope of the CHMF of traditional Chinese medicine and provide evidence supporting its safety.

$\begin{array}{ll}\text { Abbreviations } \\ \text { ICH: } & \text { Intracerebral haemorrhage } \\ \text { HICH: } & \text { Hypertensive intracerebral haemorrhage } \\ \text { RCT: } & \text { Randomized controlled trial } \\ \text { CT: } & \text { Computed tomography } \\ \text { NIHSS: } & \text { National Institutes of Health Stroke Scale } \\ \text { GCS: } & \text { Glasgow Coma Scale } \\ \text { ApoE: } & \text { Apolipoprotein E } \\ \text { TCM: } & \text { Traditional Chinese medicine } \\ \text { BI index: } & \text { Barthel index } \\ \text { FAQ: } & \text { Function activity questionnaire } \\ \text { DSM: } & \text { Data safety management } \\ \text { CHMF: } & \text { Chinese herbal medicine formula } \\ \text { DSTGP: } & \text { The Department of Science and Technology of } \\ & \text { Guangdong Province of PRC. } \\ \text { GSTIC: } & \text { The Guangzhou Science Technology and } \\ & \text { Innovation Commission of PRC. } \\ \text { GPHTCM: } & \text { The Guangdong Province Hospital of } \\ & \text { Traditional Chinese Medicine. }\end{array}$

\section{Data Availability}

The data used to support the findings of this study are available from the corresponding author upon request.

\section{Ethical Approval}

This study has been approved with ethics review No. B2016135-01 from the Institutional Ethics Committee of Guangdong Provincial Hospital of Chinese Medicine. The approvals from the subcentre ethics committees must be released before any case inclusion of this study according to their ethical review process and standards. The study initiation is on the basis of the consensus reached by the Guangdong Province Hospital of TCM and the State Administration of Traditional Chinese Medicine of the PRC both.

\section{Disclosure}

The trial was first designed on the 11th of October 2017, and subject recruitment began in February 2018. The authors are from GPHTCM and other participated research centres of this study registered on the ClinicalTrials.gov.

\section{Conflicts of Interest}

The Department of Science and Technology of Guangdong Province (DSTGP) and the Guangzhou Science Technology and Innovation Commission (GSTIC) audit and support this study. The authors declare that they have no conflicts of interest.

\section{Authors' Contributions}

Jianwen Guo was the coordinator of the study and prepared the manuscript including the trial design, quality control, and data analysis. The writing of the protocol, critical revision, and registration on ClinicalTrials.gov were done by Qixin Zhang, Jianwen Guo, and Liling Zeng. All the authors agreed on the final version of the manuscript. Liling Zeng and Dr. Qixin Zhang contributed equally to this manuscript.

\section{Acknowledgments}

Thanks are due to Professor Zehuai Wen, Dr. Xiaohui Guo, Dr. Meiling Weng, and the members from the Key Unit of Methodology in Clinical Research of Guangdong Province Hospital of Traditional Chinese Medicine (GPHTCM) (the research base), who contributed to the trial random scheme, statistical method design, and data management, providing the neuroscience assistance and all the functional assistance including methodology, data analysis, medical ethics, and quality monitoring. The authors are grateful for the research project support by the National Platform of Chinese Medicine Clinical Research and Operation, Management, Collaboration System Development (Grant no. 201407001), the Science and Technology Department of Guangdong Province of PRC (Grant no. 2014A020221074), the Guangzhou Science Technology and Innovation Commission of PRC (Grant no. 201804010178), and the Guangdong Province Hospital of Traditional Chinese Medicine (Grant no. YN6803). This report is supported by the DSTGP, the GSTIC, and the GPHTCM cooperatively and conducted by the GPHTCM as well.

\section{References}

[1] GBD 2015 Mortality and Causes of Death Collaboratiors Global. Regional, and national life expectancy, all-causes mortality, and cause-specific mortality for 249 causes of death.1980-2015:a systematic analysis for the Global Burden of Disease Study 2015[J]. Lancet.2016,388;1459-1544.

[2] W. Wang, B. Jiang, H. Sun et al., "Prevalence, Incidence, and Mortality of Stroke in China: Results from a Nationwide Population-Based Survey of 480687 Adults," Circulation, vol. 135, no. 8, pp. 759-771, 2017. 
[3] J. C. Hemphill III, S. M. Greenberg, C. Anderson et al., "Guidelines for the management of spontaneous intracerebral hemorrhage: a guideline for healthcare professionals from the American Heart Association/American Stroke Association," Stroke, vol. 46, no. 7, pp. 2032-2060, 2015.

[4] D. Rodriguez-Luna, P. Coscojuela, M. Rubiera et al., "Ultraearly hematoma growth in active intracerebral hemorrhage," Neurology, vol. 87, no. 4, pp. 357-364, 2016.

[5] Yu. Kong, Bo. Yan, Z. Guoqing et al., "Analysis of the time process and related factors of hematoma enlargement after acute cerebral hemorrhage," Jinagsu Medical Journal, vol. 41, no. 24, pp. 3032-3033, 2015.

[6] R. Wada, R. I. Aviv, A. J. Fox et al., "CT angiography "spot sign" predicts hematoma expansion in acute intracerebral hemorrhage," Stroke, vol. 38, no. 4, pp. 1257-1262, 2007.

[7] G. B. F. Porto, A. M. Spiotta, J. A. Chalela, R. T. Kellogg, and E. C. Jauch, "Blood Pressure Guideline Adherence in Patients with Ischemic and Hemorrhagic Stroke in the Neurointensive Care Unit Setting," Neurocritical Care, vol. 23, no. 3, pp. 313-320, 2015.

[8] T. Emiru, E. M. Bershad, N. D. Zantek et al., "Intracerebral hemorrhage: A review of coagulation function," Clinical and Applied Thrombosis/Hemostasis, vol. 19, no. 6, pp. 652-662, 2013.

[9] S. M. Greenberg, J. G. Vonsattel, A. Z. Segal et al., "Association of apolipoprotein E 2 and vasculopathy in cerebral amyloid angiopathy," Neurology, vol. 50, no. 4, pp. 961-965, 1998.

[10] H. B. Brouwers, A. Biffi, A. M. Ayres et al., "Apolipoprotein e genotype predicts hematoma expansion in lobar intracerebral hemorrhage," Stroke, vol. 43, no. 6, pp. 1490-1495, 2012.

[11] U. K. Misra, J. Kalita, and B. I. Somarajan, "Recurrent intracerebral hemorrhage in patients with hypertension is associated with APOE gene polymorphism: A preliminary study," Journal of Stroke and Cerebrovascular Diseases, vol. 22, no. 6, pp. 758763, 2013.

[12] T. Steiner, C. Vincent, S. Morris, S. Davis, L. Vallejo-Torres, and M. C. Christensen, "Neurosurgical outcomes after intracerebral hemorrhage: Results of the Factor Seven for Acute Hemorrhagic Stroke Trial (FAST)," Journal of Stroke and Cerebrovascular Diseases, vol. 20, no. 4, pp. 287-294, 2011.

[13] A. Saxena, C. S. Anderson, X. Wang et al., "Prognostic Significance of Hyperglycemia in Acute Intracerebral Hemorrhage," Stroke, p. STROKEAHA.115.011627.

[14] A. D. Mendelow, B. A. Gregson, E. N. Rowan, G. D. Murray, A. Gholkar, and P. M. Mitchell, "Early surgery versus initial conservative treatment in patients with spontaneous supratentorial lobar intracerebral haematomas (STICH II): a randomised trial," The Lancet, vol. 382, no. 9890, pp. 397-408, 2013.

[15] The national 8th five-year key research project, Ren, Runquan Jin, , Broken blood stasis, purging heat, Xingshen phlegm resuscitation in treatment of hemorrhagic stroke in acute stage patients with 222 focuses on clinical [C]// Research Institute of the State Administration of traditional Chinese medicine encephalopathy construction seminar and stroke research to promote the exchange of the,.

[16] C. Offiah, M. Heran, and D. Graeb, "Lightning strike: a rare cause of bilateral ossicular disruption," American Journal of Neuroradiology, vol. 28, no. 5, pp. 974-975, 2007.

[17] Yafa Xu, Jianwen Guo, Xian Liu, Juehui Li, Jing Wang, and Lingbo Hou, "Can Herbal Medicine Cause Hematoma Enlargement of Hypertensive Intracerebral Hemorrhage within $24 \mathrm{hrs}$ Time Window? A Retrospective Study of 256 Cases from a Single Center in China," Evidence-Based Complementary and Alternative Medicine, vol. 2015, Article ID 868731, 8 pages, 2015.
[18] Z. Liling, Treatment of hyperacute cerebral hemorrhage in the treatment of hyperacute cerebral hemorrhage: a multicenter randomized controlled clinical study, Guangzhou University of Chinese Medicine, Guangzhou, China, 2017.

[19] Y. Fujii, R. Tanaka, S. Takeuchi, T. Koike, T. Minakawa, and O. Sasaki, "Hematoma enlargement in spontaneous intracerebral hemorrhage," Journal of Neurosurgery, vol. 80, no. 1, pp. 51-57, 1994.

[20] Y. Fujii, S. Takeuchi, O. Sasaki, T. Minakawa, and R. Tanaka, "Multivariate analysis of predictors of hematoma enlargement in spontaneous intracerebral hemorrhage," Stroke, vol. 29, no. 6, pp. 1160-1166, 1998.

[21] K. Fujitsu, M. Muramoto, Y. Ikeda, Y. Inada, I. Kim, and T. Kuwabara, "Indications for surgical treatment of putaminal hemorrhage: Comparative study based on serial CT and timecourse analysis," Journal of Neurosurgery, vol. 73, no. 4, pp. 518$525,1990$.

[22] Neurology branch of the Chinese Medical Association. Guidelines for the diagnosis and treatment of cerebral hemorrhage in China (2014). Journal of Chinese neurology, 2015, 48 (6): 435444.

[23] X. W. Wang, "Study on Molecular Predictive Biomarkers For Early Hematoma Growth of Intracerebral hemorrhage," Chinese Journal of Stroke, 2009.

[24] A. Biffi, C. D. Anderson, J. M. Jagiella et al., "APOE genotype and extent of bleeding and outcome in lobar intracerebral haemorrhage: A genetic association study," The Lancet Neurology, vol. 10, no. 8, pp. 702-709, 2011.

[25] G. Appelboom, M. Piazza, J. E. Han et al., "Von Willebrand factor genetic variant associated with hematoma expansion after intracerebral hemorrhage," Journal of Stroke and Cerebrovascular Diseases, vol. 22, no. 6, pp. 713-717, 2013. 


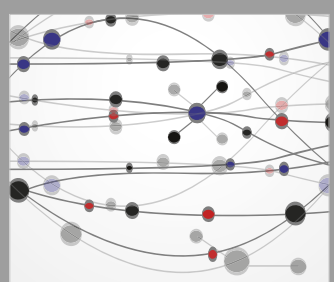

The Scientific World Journal
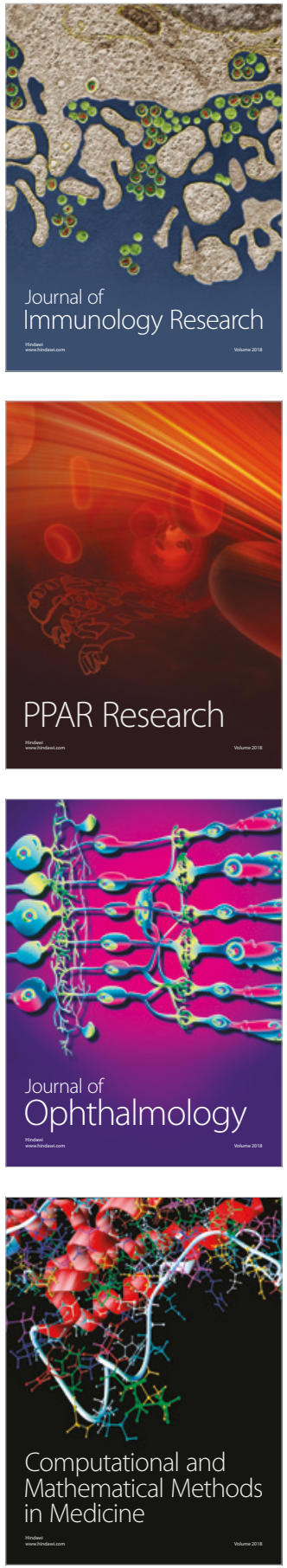

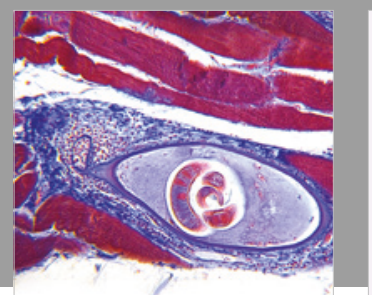

Gastroenterology Research and Practice

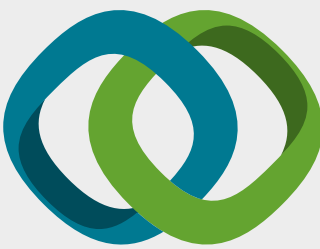

\section{Hindawi}

Submit your manuscripts at

www.hindawi.com
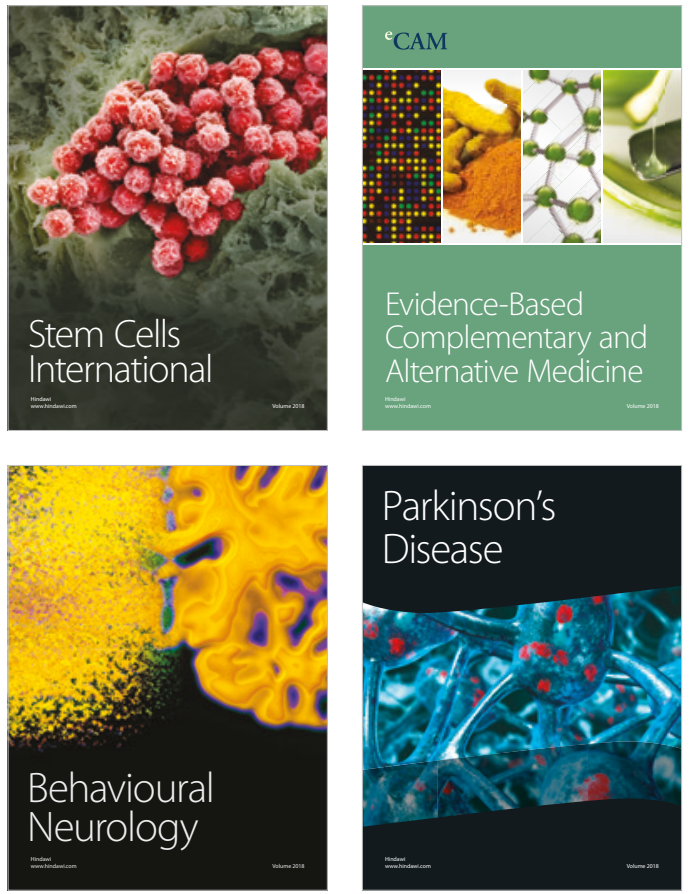

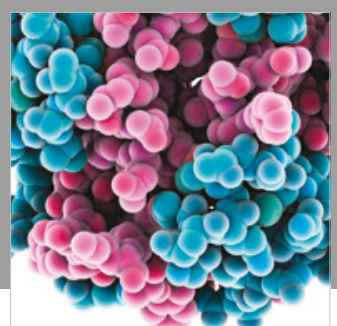

ournal of

Diabetes Research

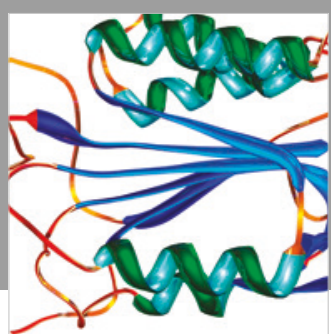

Disease Markers
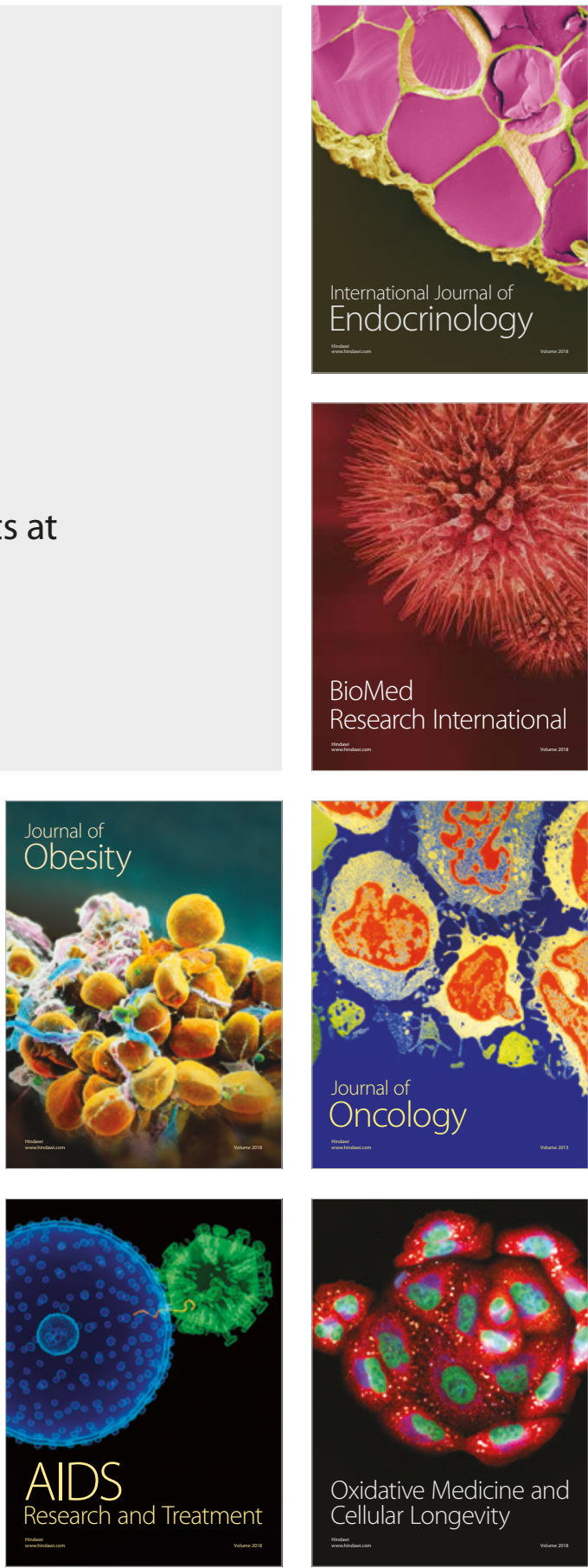